\title{
Special Issue Call for Papers: Electrophysiological Studies of Autism Spectrum Disorders
}

Guest Editors:

James C. McPartland

Raphael Bernier

Mikle South

Research employing electroencephalography, electromyography, cardiac measures, magnetoencephalography, and electrodermal response offers key insights into the neural and physiological mechanisms underlying autistic impairments in social interaction, communication, and restricted and repetitive behaviors. These methods hold promise to advance understanding of autism by elucidating biological mechanisms, by informing clinical understanding through specification of the phenotype, and by guiding intervention research through description of treatment targets.

This special issue of the Journal of Autism and Developmental Disorders (JADD) will focus on electrophysiological research in autism. The issue offers an international forum for researchers to present current scientific and methodological developments, with special emphasis on innovation across all stages of the translational science process. Reviews of extant research that offer novel theoretical or synthetic contributions are also appropriate.

Example topics include studies employing electrophysiological methods to investigate:

- Predictors of outcome and response to treatment

- Early indicators of atypical development or autism risk

- Atypical social or communicative development
- Restricted/repetitive behaviors associated with autism

- Compensatory brain functions

- Relationships among biological mechanisms, adaptive function, and/or strengths or vulnerabilities in socialcommunicative or behavioral skills

- Social cognition, including imitation, affective understanding, joint attention, empathy, theory of mind, and face perception

- Reward, attention, and learning processes, including executive function

- Sensory perception (high- and low-level) across visual, auditory, and tactile domains, as well as integration of information across sensory domains

- The broader autism phenotype

Manuscripts should be submitted by November 1, 2012 via JADD's online editorial manager (http://www.editorial manager.com/jadd/default.asp). When submitting, please designate article type, "SI: Electrophysiology of Autism." Submitted manuscripts should be prepared in accordance with JADD author guidelines for articles (20-23 manuscript pages) and should include a cover letter clarifying submission for the special issue, explaining relevance to this issue, and suggesting three referees. All manuscripts will undergo standard peer-review. Please direct any questions about scientific content to James McPartland (james. mcpartland@yale.edu), Raphael Bernier (rab2@u.washing ton.edu), or Mikle South (south@byu.edu). 